\title{
On a functional equation satisfied by certain Dirichlet series
}

by

E. Carletti and G. Monti Bragadin (Genova)

1. Introduction and notation. In [1] we obtained the meromorphic continuation of the Dirichlet series

$$
L(s)=\sum_{n=1}^{\infty} \frac{P(n)}{(n+a)^{s}(n+b)^{s}}
$$

by giving a representation of $L(s)$ in terms of Hurwitz zeta functions. That representation allowed us to get some information about zeros and poles; nevertheless no functional equation could be deduced from it. In this paper following a classical argument we obtain for $L(s)$ as above, under suitable hypothesis, a functional equation of Riemann's type. More precisely, let us consider the Dirichlet series

$$
L(s)=\sum_{n=1}^{\infty} \frac{P(n)}{(n+a)^{s}(n+b)^{s}}, \quad \operatorname{Re}(s)>\frac{d+1}{2},
$$

where $a<b$ are non-negative rational numbers and $P(X)$ is a polynomial of degree $d$ with complex coefficients with $P(0)=0$. Then by Stanley [6], Corollaries 4.5 and 4.6 , p. 115 ,

$$
G(z)=\sum_{n=1}^{\infty} P(n) z^{n}=\frac{Q(z)}{(1-z)^{d+1}},
$$

$Q(z)$ being a polynomial of degree $h=d-r$, with $r$ the greatest integer $\neq 0$ such that $P(-1)=\ldots=P(-r)=0$, and moreover, $Q(1) \neq 0, Q(0)=0$. We put $\delta=b-a, \Delta=(a+b) / 2$ and $H(z)=G\left(e^{z}\right) e^{\Delta z}$. If $\Delta=q^{\prime} / q$ (with $\left.\left(q^{\prime}, q\right)=1\right)$ then $H(z)$ is a meromorphic function of period $2 q \pi i$ with poles at $s=2 n \pi i, n \in \mathbb{Z}$. We have the Laurent expansion

$$
H(z)=\sum_{m=-(d+1)}^{\infty} \alpha_{m}^{n}(z-2 n \pi i)^{m}
$$


with $\alpha_{m}^{n}=\alpha_{m}^{n+k q}, k \in \mathbb{Z}$, and

$$
\left|\alpha_{m}^{n}\right|<B \quad \forall n, m,
$$

$B$ being a positive constant.

Let us denote by $I_{\nu}(z)$ the Bessel function defined by

$$
I_{\nu}(z)=\sum_{n=0}^{\infty} \frac{1}{n ! \Gamma(\nu+n+1)}\left(\frac{z}{2}\right)^{\nu+2 n} .
$$

Then $I_{\nu}(z)$ is holomorphic in $\mathbb{C} \backslash\{z \in \mathbb{C}: \operatorname{Re}(z) \leq 0, \operatorname{Im}(z)=0\}$ and an entire function of $\nu$. We recall the asymptotic behaviour of $I_{\nu}(z)$ (see [3], p. 962.5):

(3) $\quad I_{\nu}(z) \sim \frac{e^{z}}{\sqrt{2 \pi z}}\left(1+O\left(|z|^{-1}\right)\right)+\frac{e^{-z \pm(\nu+1 / 2) \pi i}}{\sqrt{2 \pi z}}\left(1+O\left(|z|^{-1}\right)\right), \quad|z| \rightarrow \infty$ (the $+\operatorname{sign}$ is taken for $\pi / 2<\arg z<3 \pi / 2$ and the $-\operatorname{sign}$ for $-3 \pi / 2<$ $\arg z<\pi / 2)$, and the relations

$$
I_{\nu}\left(e^{\pi m i} z\right)=e^{\nu \pi m i} I_{\nu}(z), \quad m \in \mathbb{Z}
$$

(see [3], 8.476, n. 4, p. 968),

$$
\frac{d^{p}}{d z^{p}} z^{\nu} I_{\nu}(z)=z^{\nu} I_{\nu-p}(z)
$$

(see [3], 8.486, n. 5, p. 970).

In this paper we prove the following

THEOREM. With the above notation and hypothesis, if $h+b \leq d+1$, then $L(s)$ has a meromorphic continuation onto $\mathbb{C}$ with at most simple poles at $s=(d-l+1) / 2, l=0,1, \ldots$ and satisfies the functional equation

$$
\xi(s)=-\xi(1-s)
$$

where

and for $\operatorname{Re}(s)>1$

$$
\xi(s)=\delta^{s-1 / 2} \Phi_{L}(s) \Gamma(s) L(s)
$$

$$
\begin{aligned}
\Phi_{L}(s)= & \left(\frac{\delta}{2}\right)^{s-1 / 2} \sum_{t=1}^{q} \sum_{p=0}^{d} \frac{1}{p !}\left(\frac{\delta}{2}\right)^{p} \alpha_{-p-1}^{t} \\
& \times \sum_{n \in \mathbb{Z}}^{*}(2(n q+t) \pi i)^{1 / 2-s} I_{1 / 2-s-p}(\delta(n q+t) \pi i)
\end{aligned}
$$

(* means that if $t=q$ then $n \neq-1$ ).

The Theorem above has an interesting application to Minakshisundaram-Pleijel zeta functions of the real spheres and real and complex projective spaces. The problem of finding the functional equation for such zeta functions goes back to Minakshisundaram and Pleijel (see [4], [5] and [2]). 
Corollary. Let

$$
Z\left(\mathbb{S}^{k}, s\right)=\frac{1}{(k-1) !} \sum_{n=1}^{\infty} \frac{(n+1) \ldots(n+k-2)(2 n+k-1)}{n^{s}(n+k-1)^{s}}
$$

be the Minakshisundaram-Pleijel zeta function of sphere $\mathbb{S}^{k}$. Then $Z\left(\mathbb{S}^{k}, s\right)$ satisfies the functional equation

$$
Z\left(\mathbb{S}^{k},-s\right)=-Z\left(\mathbb{S}^{k}, s-1\right) \frac{\Phi_{L}(s) \Gamma(s)}{\Phi_{L}(1-s) \Gamma(1-s)}(k-1)^{2 s-1}
$$

where $L(s+1)=Z\left(\mathbb{S}^{k}, s\right)$.

For the real projective space $\mathbb{P}^{k}(\mathbb{R})$ and the associated Minakshisundaram-Pleijel zeta function

$$
Z\left(\mathbb{P}^{k}(\mathbb{R}), s\right)=\frac{1}{(k-1) !} \sum_{n=1}^{\infty} \frac{(2 n+1) \ldots(2 n+k-2)(4 n+k-1)}{(2 n)^{s}(2 n+k-1)^{s}}
$$

we have

$$
Z\left(\mathbb{P}^{k}(\mathbb{R}),-s\right)=-Z\left(\mathbb{P}^{k}(\mathbb{R}), 1-s\right) \frac{\Phi_{L}(s) \Gamma(s)}{\Phi_{L}(1-s) \Gamma(1-s)}\left(\frac{k-1}{2}\right)^{2 s-1},
$$

where $L(s+1)=Z\left(\mathbb{P}^{k}(\mathbb{R}), s\right)$.

For the complex projective space $\mathbb{P}^{k}(\mathbb{C})$ and the associated Minakshisundaram-Pleijel zeta function

$$
Z\left(\mathbb{P}^{k}(\mathbb{C}), s\right)=\frac{1}{((k-1) !)^{2}} \sum_{n=1}^{\infty} \frac{((n+1) \ldots(n+k-2))^{2}(2 n+k) k}{(4 n)^{s}(n+k)^{s}}
$$

we have

$$
Z\left(\mathbb{P}^{k}(\mathbb{C}),-s\right)=-Z\left(\mathbb{P}^{k}(\mathbb{C}), 1-s\right) \frac{\Phi_{L}(s) \Gamma(s)}{\Phi_{L}(1-s) \Gamma(1-s)}(k-1)^{2 s-1},
$$

where $L(s+1)=Z\left(\mathbb{P}^{k}(\mathbb{C}), s\right)$.

2. Two lemmas. Starting from the classical formula

$$
\Gamma(s)=\int_{0}^{\infty} e^{-t} t^{s-1} d t
$$

one easily gets

$$
\Gamma(s)^{2} L(s)=\int_{0}^{\infty} \int_{0}^{\infty} G\left(e^{-\left(t_{1}+t_{2}\right)}\right) e^{-a t_{1}-b t_{2}}\left(t_{1} t_{2}\right)^{s-1} d t_{1} d t_{2} .
$$

Using the substitution

$$
\left\{\begin{array}{l}
t_{1}=t u, \\
t_{2}=t(1-u), \quad 0 \leq t \leq \infty, 0 \leq u \leq 1,
\end{array}\right.
$$


we obtain

$$
\Gamma(s)^{2} L(s)=\int_{0}^{\infty} G\left(e^{-t}\right) e^{-b t} t^{2 s-1} \int_{0}^{1} u^{s-1}(1-u)^{s-1} e^{\delta t u} d u d t .
$$

Now, by [3], n. 3382.2 , p. 319 we have

$$
\frac{1}{\sqrt{\pi}} \delta^{s-1 / 2} \Gamma(s) L(s)=\int_{0}^{\infty} G\left(e^{-t}\right) e^{-\Delta t} I_{s-1 / 2}\left(\frac{1}{2} \delta t\right) t^{s-1 / 2} d t .
$$

Lemma 1. Define

$$
I(s)=\frac{1}{2 \pi i} \int_{C} G\left(e^{z}\right) e^{\Delta z} I_{s-1 / 2}\left(\frac{1}{2} \delta z\right) z^{s-1 / 2} d z,
$$

where $C=C_{1} \cup C_{2} \cup C_{3}$ and $C_{1}=\left\{z \in \mathbb{C}: z=r e^{-\pi i}, r \in(\varrho, \infty)\right\}$, $C_{3}=\left\{z \in \mathbb{C}: z=r e^{\pi i}, r \in(\varrho, \infty)\right\}, C_{2}=\left\{z \in \mathbb{C}: z=\varrho e^{\theta i},-\pi \leq \theta \leq \pi\right\}$ with $0<\varrho<2 \pi$ ( $C$ is counter-clockwise oriented). Then $I(s)$ is well defined (independent of $\varrho$ ) and entire. Furthermore, we have

$$
I(s)=\frac{1}{\pi \sqrt{\pi}} \delta^{s-1 / 2} \Gamma(s) L(s) \sin 2 \pi s \quad \forall s \in \mathbb{C} .
$$

Proof. We need to prove that our integral in (2) is uniformly and absolutely convergent on compact subsets of $\mathbb{C}$. The convergence along $C_{2}$ is trivial. If $z \in C_{1} \cup C_{3}$ then $\operatorname{Re}(z)=-r$ and $e^{z}=e^{-r}$ so that for $r \geq 1$ we have

$$
\left|z^{s-1 / 2}\right| \leq r^{M-1 / 2} e^{\pi M} \quad \text { if }|s|<M,
$$

and by (3),

$$
\begin{aligned}
\left|G\left(e^{z}\right) e^{\Delta z} I_{s-1 / 2}(\delta z / 2)\right| & \sim\left|\frac{Q\left(e^{z}\right)}{\left(1-e^{z}\right)^{d+1}} e^{\Delta z} \frac{e^{\delta z / 2}+e^{-\delta z / 2 \pm s \pi i}}{\sqrt{\pi \delta z}}\left(1+O\left(|z|^{-1}\right)\right)\right| \\
& =O\left(e^{-r}\right),
\end{aligned}
$$

so the first statement follows.

We have

where

$$
I(s)=\frac{1}{2 \pi i}\left(\int_{C_{1}}+\int_{C_{2}}+\int_{C_{3}}\right) G\left(e^{z}\right) e^{\Delta z} I_{s-1 / 2}(\delta z / 2) z^{s-1 / 2} d z,
$$

$$
\int_{C_{1}}=\int_{\infty}^{\varrho} G\left(e^{-r}\right) e^{-\Delta r} I_{s-1 / 2}\left(\delta r e^{-\pi i} / 2\right) r^{s-1 / 2} e^{-\pi i(s-1 / 2)} e^{-\pi i} d r
$$

since $d z=e^{-\pi i} d r$ and

$$
\int_{C_{3}}=\int_{\varrho}^{\infty} G\left(e^{-r}\right) e^{-\Delta r} I_{s-1 / 2}\left(\delta r e^{\pi i} / 2\right) r^{s-1 / 2} e^{\pi i(s-1 / 2)} e^{\pi i} d r
$$

since $d z=e^{\pi i} d r$. 
By (4) we obtain

$$
\int_{C_{1}}+\int_{C_{3}}=2 i \sin 2 \pi s \int_{\varrho}^{\infty} G\left(e^{-r}\right) e^{-\Delta r} I_{s-1 / 2}(\delta r / 2) r^{s-1 / 2} d r .
$$

Along $C_{2}, z=\varrho e^{i \theta}$, and

$$
\int_{C_{2}}=\int_{-\pi}^{\pi} F(\theta, \varrho) \varrho^{s-1 / 2} d \theta
$$

where $F(\theta, \varrho)$ is uniformly bounded, with respect to $\varrho$. Then

$$
\lim _{\varrho \rightarrow 0} \int_{C_{2}}=0
$$

if $\operatorname{Re}(s)$ is sufficiently large and so we have

$$
I(s)=\frac{1}{\pi} \sin 2 \pi s \int_{0}^{\infty} G\left(e^{-r}\right) e^{-\Delta r} I_{s-1 / 2}(\delta r / 2) r^{s-1 / 2} d r
$$

so that

$$
I(s)=\frac{1}{\sqrt{\pi^{3}}} \delta^{s-1 / 2} L(s) \Gamma(s) \sin 2 \pi s .
$$

The above identity holds on the whole plane by analytic continuation.

Lemma 2. With the above notation if $h+b \leq d+1$ and $\operatorname{Re}(s)>1$ we have

$$
\begin{aligned}
I(1-s)= & (\delta / 2)^{s-1 / 2} \sum_{t=1}^{q} \sum_{p=0}^{d} \frac{1}{p !}(\delta / 2)^{p} \alpha_{-p-1}^{t} \\
& \times \sum_{n \in \mathbb{Z}}^{*}(2(n q+t) \pi i)^{1 / 2-s} I_{1 / 2-s-p}(\delta(n q+t) \pi i) .
\end{aligned}
$$

Proof. Let $N$ be an odd integer and define

$$
I_{N}(s)=\frac{1}{2 \pi i} \int_{C_{N}} G\left(e^{z}\right) e^{\Delta z} I_{s-1 / 2}(\delta z / 2) z^{s-1 / 2} d z,
$$

where $C_{N}=\{z:|z|=\varrho\} \cup\{z:|z|=N \pi\} \cup\left\{z: z=r e^{\pi i}, 0<\varrho \leq r \leq\right.$ $N \pi\} \cup\left\{z: z=r e^{-\pi i}, 0<\varrho \leq r \leq N \pi\right\}\left(C_{N}\right.$ is oriented in such way that $\{z:|z|=\varrho\}$ is counter-clockwise oriented). We see that $I_{N}(s) \rightarrow I(s)$ as $N \rightarrow \infty$ if $\sigma=\operatorname{Re}(s)<0$. In fact, on $|z|=N \pi$ we have

$$
\begin{gathered}
\left|z^{s-1 / 2}\right| \leq(N \pi)^{\sigma-1 / 2} e^{\pi|t|} \\
\left|H(z) I_{s-1 / 2}(\delta z / 2)\right| \leq A_{1} N^{-1 / 2}\left(1+e^{\pi|t|}\right)\left(1+O\left(N^{-1}\right)\right)
\end{gathered}
$$

with $A_{1}$ a suitable positive constant by (3), so that 


$$
\left|z^{s-1 / 2} G\left(e^{z}\right) e^{\Delta z} I_{s-1 / 2}(\delta z / 2)\right| \leq N^{\sigma-1} e^{2 \pi|t|} A_{2}
$$

with $A_{2}$ a suitable positive constant depending on $\sigma$. Hence

if $\sigma<0$.

$$
\lim _{N \rightarrow \infty} \int_{|z|=N \pi}=0
$$

By Cauchy's theorem we have

$$
I_{N}(s)=\sum_{-N \leq 2 n \leq N} \operatorname{Res}\left(G\left(e^{z}\right) e^{\Delta z} I_{s-1 / 2}(\delta z / 2) z^{s-1 / 2} ; 2 \pi n i\right) .
$$

Put

$$
A(z)=I_{s-1 / 2}(\delta z / 2) z^{s-1 / 2}
$$

and consider its Taylor series at $s=2 n \pi i, n \neq 0$ :

Then we have

$$
A(z)=\sum_{m=0}^{\infty} \frac{1}{m !} A^{(m)}(2 n \pi i)(z-2 n \pi i)^{m} .
$$

$$
\operatorname{Res}(H(z) A(z) ; 2 \pi n i)=\sum_{\substack{p+l=-1 \\ p \geq-(d+1) \\ l \geq 0}} \frac{1}{l !} \alpha_{p}^{n} A^{(l)}(2 n \pi i)=\sum_{p=0}^{d} \frac{1}{p !} \alpha_{-p-1}^{n} A^{(p)}(2 n \pi i) .
$$

By (5),

$$
A^{(p)}(z)=z^{s-1 / 2} I_{s-1 / 2-p}(\delta z / 2)(\delta / 2)^{1 / 2-s+p} .
$$

Therefore

$$
I_{N}(s)=\sum_{-N \leq 2 n \leq N} \sum_{\substack{n \neq 0 \\ p=0}}^{d} \frac{1}{p !} \alpha_{-p-1}^{n}(2 n \pi i)^{s-1 / 2} I_{s-1 / 2-p}(\delta n \pi i)(\delta / 2)^{1 / 2-s+p} .
$$

Because of (3) and (2) the series

$$
\sum_{n \neq 0} \alpha_{-p-1}^{n}(2 n \pi i)^{1 / 2-s} I_{1 / 2-s-p}(\delta n \pi i)
$$

converges absolutely and uniformly on compact subsets of $\sigma>1$. Thus for $\sigma>1$, we have

$$
\begin{aligned}
I(1-s)= & \sum_{\substack{n \in \mathbb{Z} \\
n \neq 0}} \sum_{p=0}^{d} \frac{1}{p !} \alpha_{-p-1}^{n}(2 n \pi i)^{1 / 2-s} I_{1 / 2-s-p}(\delta n \pi i)(\delta / 2)^{s-1 / 2+p} \\
= & (\delta / 2)^{s-1 / 2} \sum_{t=1}^{q} \sum_{p=0}^{d} \frac{1}{p !}(\delta / 2)^{p} \alpha_{-p-1}^{t} \\
& \times \sum_{n \in \mathbb{Z}}^{*}(2(n q+t) \pi i)^{1 / 2-s} I_{1 / 2-s-p}(\delta(n q+t) \pi i) .
\end{aligned}
$$




\section{Proof of the Theorem and the Corollary}

Proof of Theorem. Put $\Phi_{L}(s)=I(1-s)$. By (6) we have

$$
L(s)=\sqrt{\pi} \delta^{1 / 2-s} \Gamma(1-s) \frac{1}{2 \cos \pi s} \Phi_{L}(1-s) ;
$$

so if we put

$$
\xi(s)=\delta^{s-1 / 2} \Phi_{L}(s) \Gamma(s) L(s)
$$

then $\xi(s)=-\xi(1-s)$ and the Theorem is proved.

Proof of Corollary. Consider

$$
L_{k}(s)=\frac{1}{(k-1) !} \sum_{n=1}^{\infty} \frac{n \ldots(n+k-1)(2 n+k-1)}{n^{s}(n+k-1)^{s}} .
$$

Then $Z_{k}\left(\mathbb{S}^{k}, s\right)=L_{k}(s+1)$. Furthermore, $L_{k}(s)$ satisfies the hypothesis of the Theorem and so

$$
L_{k}(1-s)=-L_{k}(s) \frac{\Phi(s) \Gamma(s)}{\Phi(1-s) \Gamma(1-s)}(k-1)^{2 s-1} .
$$

A similar argument works for projective spaces and the Corollary follows.

R e mark. If $a=b$ we obtain, by using the same method, a simpler integral representation for $L(s)$. In particular, we get $\xi(s)=\Phi_{L}(s) \Gamma(2 s) L(s)$ where, for $\operatorname{Re}(s)>1$,

$$
\begin{aligned}
\Phi_{L}(s)= & \sum_{t=1}^{q} \sum_{p=0}^{d} \frac{1}{p !}\left(\begin{array}{c}
1-2 s \\
p
\end{array}\right) \alpha_{-p-1}^{t} \sum_{n \in \mathbb{Z}}^{*}(2 \pi i(n q+t))^{1-2 s-p} \\
= & 2 \sum_{t=1}^{q} \sum_{p=0}^{d} \frac{1}{p !}\left(\begin{array}{c}
1-2 s \\
p
\end{array}\right) \alpha_{-p-1}^{t}(2 \pi q)^{1-p-2 s} \\
& \times \cos \frac{\pi}{2}(1-p-2 s) \zeta(2 s+p-1, t / q)
\end{aligned}
$$

where $\zeta(s, a)$ is the Hurwitz zeta function.

\section{References}

[1] E. Carletti and G. Monti Bragadin, On Dirichlet series associated with polynomials, Proc. Amer. Math. Soc. 121 (1994), 33-37.

[2] -, -, On Minakshisundaram-Pleijel zeta functions of spheres, ibid. 122 (1994), 993-1001.

[3] I. S. Gradshteyn and I. M. Ryzhik, Tables of Integrals, Series and Products, Academic Press, 1980.

[4] S. Minakshisundaram, Zeta functions on the sphere, J. Indian Math. Soc. 13 (1949), 41-48. 
[5] S. Minakshisundaram and A. Pleijel, Some properties of the eigenfunctions of the Laplace operator on Riemannian manifolds, Canad. J. Math. 1 (1949), 242-256.

[6] R. Stanley, Generating functions, in: Studies in Combinatorics, G. C. Rota (ed.), Stud. Math. 17, Math. Assoc. Amer., 1978, 100-141.

DIPARTIMENTO DI MATEMATICA

UNIVERSITÀ DI GENOVA

VIA L. B. ALBERTI 4

16132 GENOVA, ITALY

E-mail: CARLETTI@DIMA.UNIGE.IT 